\title{
Análise da flexibilidade segmentar e prevalência de lesões no futebol segundo faixa etária
}

\author{
Analysis of the joint flexibility and prevalence of soccer injuries according to age \\ Análisis de la flexibilidad segmentaria y prevalencia de lesiones en el fútbol según franja etárea \\ Danielle Almeida de Sena', Francislayni Marchiori Ferreira', Renata Helena Galvão de Melo', \\ Charles Taciro², Rodrigo Luiz Carregaro ${ }^{3}$, Silvio Assis de Oliveira Júnior ${ }^{2}$
}

RESUMO I Retrações musculares têm apresentado relações com má postura e Lesões Desportivas (LD) em jogadores de futebol jovens e adultos. Este estudo teve por objetivo avaliar a flexibilidade articular, extensibilidade muscular e prevalência de LD em praticantes de futebol, relacionando-as com a faixa etária. A casuística integrou 170 participantes do sexo masculino, procedentes das equipes de base profissionalizante e profissional de um clube desportivo de Campo Grande (MS). Os participantes foram distribuídos em três grupos: G1 (infantojuvenil), G2 (adolescentes) e G3 (adultos). Para a tomada de informações sobre lesões, utilizou-se de um inquérito de morbidade referida. Foram realizadas análises para caracterização antropométrica, flexibilidade articular, extensibilidade muscular e alinhamento corporal. Em relação à prevalência de LD, foram registrados 55 LD durante as duas últimas temporadas, sendo que 48 participantes (28,23\%) relataram presença de LD. O G3 revelou a maior taxa de prevalência de LD, totalizando 0,68 LD/atleta. A taxa de lesão por atleta lesionado apresentou-se maior no G2, com 1,4 LM/atleta lesionado. O G2 apresentou maior grau de flexibilidade articular do quadril, com alcance de 26,3 $\pm 8,0 \mathrm{~cm}$ no teste de sentar e alcançar. O G3 apresentou os maiores índices de prevalência de retração para flexores de quadril. Ao teste de Schöber, o G2 mostrou a maior prevalência de inflexibilidade lombar. Conclui-se que atletas profissionais têm maior incidência e prevalência de LD. Os índices de inflexibilidade foram particularmente importantes em faixas etárias mais jovens, sugerindo que uma possível interação entre atributos intrínsecos e extrínsecos se associe a distúrbios musculoesqueléticos em jovens atletas.

Descritores | Músculo Esquelético; Traumatismos em Atletas; Futebol; Grupos Etários.
ABSTRACT I Muscle shortening has been associated with asymmetrical posture and Sports Injuries (SI) in soccer players in distinct ages. The objective of the present study was to analyze the joint flexibility, muscle extensibility and the SI prevalence in soccer players according to age: young and adults practitioners. Studied subjects integrated 170 male soccer players from amateurs and professional teams of a sports club from Campo Grande (MS), Brazil. Participants were divided into three age groups: G1 (juvenile), G2 (teenagers) and G3 (adults). To obtain information about injuries, was used a morbidity survey. Anthropometry and clinical tests were performed to analyze the joint flexibility (sit and reach test) and muscle extensibility (Thomas test; Schöber test and posture analysis). In relation to SI prevalence, 48 athletes (28.2\%) reported $\mathrm{SI}$ incidence during two last years, with register of 55 SI; G3 presented 0.68 IS/ athlete, while G2 showed 1.4 IS/injured athlete. Moreover, G2 reported higher degrees of hip flexibility, with range of $26.3 \pm 8.0 \mathrm{~cm}$ in sit and reach test, and presence of lumbar shortening in response to Schöber test. In addition, G3 exhibited greater indexes of muscle shortening in hip flexors, evidenced in Thomas examination. In conclusion, evidences have been showing that professional athletes have presented higher incidence and prevalence of sports injuries. However, muscle shortening scores were more important results in youth soccer players, suggesting a possible interaction between intrinsic and extrinsic as cause of skeletal muscle disturbances in youth athletes.

Keywords I Muscle, Skeletal; Athletic Injuries; Soccer; Age Groups.

Estudo realizado no Centro Esportivo Nova Esperança - Campo Grande (MS), Brasil.

${ }^{1}$ Curso de Fisioterapia, Centro de Ciências Biológicas e da Saúde da Universidade Federal de Mato Grosso do Sul (UFMS) - Campo Grande (MS), Brasil.

${ }^{2}$ Centro de Ciências Biológicas e da Saúde da UFMS - Campo Grande (MS), Brasil.

${ }^{3}$ Curso de Fisioterapia da Universidade de Brasília (UnB) - Brasília (DF), Brasil.

Endereço para correspondência: Silvio Assis de Oliveira Júnior - Curso de Fisioterapia da Universidade Federal de Mato Grosso do Sul, s/n, Universitário - Cidade Universitária; CEP: 79070-900 - Campo Grande (MS), Brasil - E-mail: oliveirajr.ufms@gmail.com

Apresentação: mar. 2013 - Aceito para publicação: out. 2013 - Fonte de financiamento: nenhuma - Conflito de interesses: nada a declarar - Parecer de aprovação no

Comitê de Ética no 2035 CAAE 0093.0.049.000-11. 
RESUMEN I Retracciones musculares han presentado relaciones con mala postura y Lesiones Deportivas (LD) en jugadores de fútbol jóvenes y adultos. Este estudio tuvo por objetivo evaluar la flexibilidad articular, extensibilidad muscular y prevalencia de LD en practicantes de fútbol, relacionándolas con la franja etárea. La casuística integró 170 participantes del sexo masculino, procedentes de los equipos de base profesionalizante y profesional de un club deportivo de Campo Grande (MS). Los participantes fueron distribuidos en tres grupos: G1 (infanto-juvenil), G2 (adolescentes) y G3 (adultos). Para la obtención de informaciones sobre lesiones, se utilizó una averiguación de morbilidad referida. Fueron realizados análisis para caracterización antropométrica, flexibilidad articular, extensibilidad muscular y alineamiento corporal. En relación a la prevalencia de LD, fueran registrados 55 LD durante las dos últimas temporadas, siendo que 48 participantes (28,23\%) relataron presencia de LD. El
G3 reveló la mayor tasa de prevalencia de LD, totalizando 0,68 LD/ atleta. La tasa de lesión por atleta se presentó mayor en el G2, con 1,4 LM/atleta lesionado. El G2 presentó mayor grado de flexibilidad articular de la cadera, con alcance de $26,3 \pm 8,0 \mathrm{~cm}$ en el test de sentarse y alcanzar. El G3 presentó los mayores índices de prevalencia de retracción para flexores de la cadera. Al test de Schöber, el G2 mostró la mayor prevalencia de inflexibilidad lumbar. Se concluye que atletas profesionales tienen mayor incidencia y prevalencia de LD. Los índices de inflexibilidad fueron particularmente importantes en franjas etáreas más jóvenes, sugiriendo que una posible interacción entre atributos intrínsecos y extrínsecos se asocie a trastornos musculoesqueléticos en jóvenes atletas.

Palabras clave I Músculo Esquelético; Traumatismos en Atletas; Fútbol; Grupos de Edad.

\section{INTRODUÇÃO}

O futebol tem precocemente congregado crianças e jovens na busca de desenvolvimento físico, técnico e tático, visando uma oportunidade de profissionalização desportiva ${ }^{1,2}$. Este fato tem importante relevância epidemiológica, devido aos altos riscos de Lesões Desportivas (LD) em jovens atletas ${ }^{3}$. A prática de futebol se associa a contatos físicos e gestos específicos, como corrida, saltos, aterrissagens, aceleração, desaceleração, mudanças abruptas de direção, chutes e pivoteios ${ }^{4,5}$. Contudo, demandas físicas específicas, preparo físico inadequado e alto volume de treinamento podem predispor os praticantes a $\mathrm{LD}^{6}$. Ademais, a susceptibilidade a LD é proporcional ao nível de exigência competitiva do futebol: atletas adultos e profissionais são mais propensos a LD do que atletas jovens ${ }^{3,7}$.

Além de fatores de treinamento, características do atleta, como idade ${ }^{7}$ e flexibilidade articular $^{8}$, são potenciais fatores de risco intrínseco para $\mathrm{LD}^{6}$. A flexibilidade e a força muscular são atributos físicos essenciais na execução de gestos desportivos, sofrendo adaptações com a modalidade ${ }^{9}$. Em particular, a flexibilidade é também influenciada pela faixa etária, reduzindo-se com a idade ${ }^{10}$. Por sua vez, a instalação de retrações musculares tem apresentado relações com má postura, contribuindo para a LD em jogadores jovens ${ }^{3,11}$ e adultos ${ }^{8,12}$.

Alguns trabalhos têm atribuído à postura o maior potencial etiológico para a instalação de LD em futebolistas ${ }^{13,14}$, sugerindo que há participação de desalinhamentos entre cadeias musculares. Ribeiro et al. ${ }^{13}$ justificam a importância da investigação postural como característica intrínseca a jovens atletas. Contudo, não foram encontrados estudos que tenham abordado qual desses componentes etiológicos, flexibilidade segmentar e/ou alinhamento postural global, se associa mais diretamente com a incidência de LD no futebol, em diversas idades. Este estudo teve por objetivo avaliar a flexibilidade articular, extensibilidade muscular e prevalência de LD em praticantes de futebol, relacionando-as com a idade. Outro propósito foi analisar a associação entre tais características e a incidência de LD, buscando-se identificar qual(is) o(s) fator(es) etiológico(s) de maior contribuição para a instalação de LD no futebol. Tem-se como hipótese inicial que desportistas adultos apresentam maior prevalência de retrações segmentares, que estão mais diretamente associadas com a incidência de LD.

\section{METODOLOGIA}

\section{Participantes}

Participaram do estudo 170 jogadores de futebol do sexo masculino, procedentes de um clube desportivo de Campo Grande (MS). Todos os participantes ou os seus responsáveis foram informados sobre os objetivos da pesquisa, bem como do caráter voluntário da participação, e assinaram o Termo de Consentimento Livre e Esclarecido, aprovado pelo Comitê de Ética em Pesquisa da UFMS (protocolo no 2035).

Os voluntários foram distribuídos em três grupos etários $^{15}$. O grupo 1 (G1) integrou jovens de 11 a 14 anos; o grupo 2 (G2) possuía jogadores com 15 a 18 anos completos e o grupo 3 (G3) detinha atletas com mais de 18 anos.

Os participantes foram entrevistados para obtenção de dados pessoais, histórico de prática e ocorrência de LD nas 
duas últimas temporadas. Foram feitas análises para caracterização antropométrica, flexibilidade articular, extensibilidade muscular e alinhamento corporal. Para fins de estudo, a extensibilidade muscular foi definida como a amplitude na qual a articulação pode ser movida passivamente, considerando-se a influência do comprimento muscular ${ }^{16,17}$.

Para a análise da flexibilidade do quadril e da coluna lombar, considerou-se, respectivamente, o teste de sentar e alcançar ${ }^{18}$ e o teste de Schöber modificado ${ }^{19}$. A extensibilidade dos músculos flexores do quadril foi analisada com o teste de Thomas ${ }^{20,21}$. Para a análise das cadeias musculares estáticas posterior (Postura 1) e anterior (Postura 2), foram consideradas avaliações posturais específicas $^{20,21}$. A presença de retração muscular foi considerada frente desordens compensatórias específicas ${ }^{20,21}$. Todos os procedimentos foram realizados por dois pesquisadores treinados. Para a tomada de informações sobre LD, utilizou-se de um Inquérito de Morbidade Referida $^{22}$. O perfil nosográfico dos agravos obtidos foi divulgado em recente publicação científica ${ }^{23}$.

Para a análise dos dados relativos ao perfil demográfico e histórico de treinamento, utilizou-se de análise de variância e teste de Student-Newman-Keuls. Para a análise dos resultados de avaliação física, considerou-se o teste de Goodman $^{25}$. Todas as conclusões foram discutidas para 5\% de significância estatística. $\mathrm{Na}$ vigência de significância estatística calculou-se o risco relativo (odds ratio), por meio da análise da associação entre variáveis em estudo.

\section{RESULTADOS}

Em geral, estatura, massa corporal e tempo de prática de futebol ampliaram-se com a idade, mostrando-se diferentes em todas as comparações efetivadas (Tabela 1). Foram registradas $55 \mathrm{LD}$, sendo que 48 participantes $(28,23 \%)$ relataram presença de $\mathrm{LD}$. O G3 mostrou a maior prevalência de $\mathrm{LD}$, portando 15 atletas lesionados (51,7\%), o que totalizou um índice de incidência de 0,68 LD/atleta. A taxa de LD por atleta lesionado foi maior no G2.

Em relação à flexibilidade articular do quadril, o G2 apresentou maior grau de flexibilidade, com alcance de $26,3 \pm 8,0 \mathrm{~cm}$ (Figura 1).

Quanto aos resultados de extensibilidade muscular de flexores do quadril, foram constatadas alterações em todos os grupos (Tabela 2). Considerando-se a idade, o G3 apresentou a maior prevalência de retração para flexores de quadril (97 a 100\%). Ao teste de Schöber, o G2 mostrou a maior prevalência de retração lombar. A presença de retração
Tabela 1. Características gerais segundo faixa etária

\begin{tabular}{lccc} 
& \multicolumn{3}{c}{ Grupos } \\
\cline { 2 - 4 } Variáveis & G1 & G2 & G3 \\
Idade (anos) & $12,5 \pm 1,2$ & $15,6 \pm 0,8^{*}$ & $24,4 \pm 4,7^{* \#}$ \\
Estatura (cm) & $152 \pm 20,2$ & $171 \pm 8,0^{*}$ & $177 \pm 8,0^{*}$ \\
MC (kg) & $45 \pm 11,2$ & $64 \pm 9,1^{*}$ & $74 \pm 6,2^{* \#}$ \\
HT (anos) & $3,70 \pm 2,31$ & $5,30 \pm 2,89^{*}$ & $13 \pm 4,68^{* *}$ \\
Indivíduos & 97 & 44 & 29 \\
ATL & $28(28,9 \%)$ & $5(10,41 \%)$ & $15(31,25 \%)$ \\
Lesões & $28(50,90 \%)$ & $7(12,72 \%)$ & $20(36,36 \%)$ \\
TLA & 0,29 & 0,15 & 0,68 \\
TLAL & 1 & 1,4 & 1,3
\end{tabular}

Variáveis demográficas e Histórico de Treinamento (HT) expressos em média desvio-padrão: G1: atletas da faixa etária de 11 a 14 anos de idade; G2: atletas da faixa etária de 15 a 18 anos; G3 atletas adultos; " $p<0,05$ versus $\mathrm{G1}$; " $p<0,05$ versus $\mathrm{G} 2$; $\mathrm{tp}<0,05$ versus $\mathrm{G} 3$; ANOVA e teste de Student-Newman-Keuls $(p<0,05)$. ATL: Número de Atletas Lesionados; Lesões: número total de lesões por faixa etária; TAL: Taxa de Lesão por Atleta Lesionado; TLA: Taxa de Lesão por Atleta

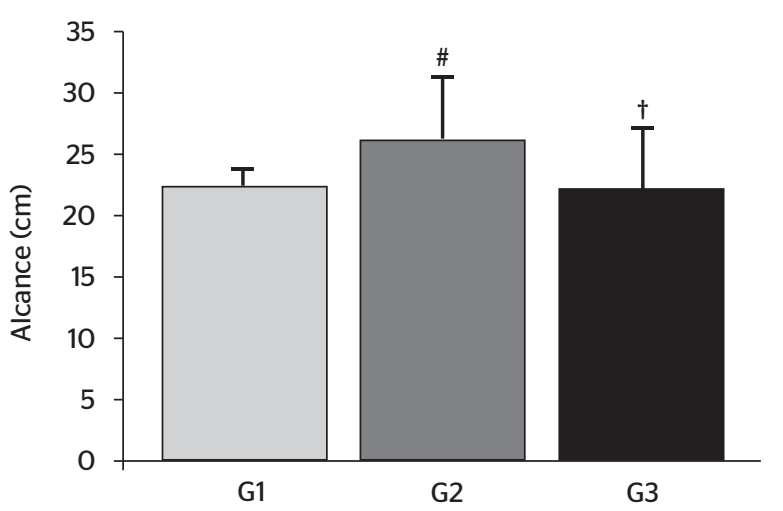

G1: atletas da faixa etária de 11 a 14 anos de idade; G2: atletas da faixa etária de 15 a 18 anos; G3: atletas adultos: $" \mathrm{p}<0,05$ versus $\mathrm{G}$; ; $p<0,05$ versus $\mathrm{G} 2$

Figura 1. Média e desvio-padrão das respostas de amplitude segmentar (cm) obtidos por meio do teste de alcance com banco de Wells

Tabela 2. Distribuição absoluta e relativa (\%) de alterações de flexibilidade segundo faixa etária

\begin{tabular}{|c|c|c|c|c|c|c|}
\hline \multirow{2}{*}{\multicolumn{2}{|c|}{ Variáveis }} & \multirow{2}{*}{ Casos } & \multicolumn{3}{|c|}{ Grupos } & \multirow{2}{*}{ Tota } \\
\hline & & & G1 & G2 & G3 & \\
\hline \multirow{4}{*}{ TD } & \multirow{2}{*}{ FM } & Ausente (-) & $81(65,9)$ & $42(34,1)^{\star}$ & $O(O, O)^{* \#}$ & 123 \\
\hline & & Presente $(+)$ & $16(34,0)^{\S}$ & $2(4,3)^{\star \S}$ & $29(61,7)^{\# \S}$ & 47 \\
\hline & \multirow{2}{*}{ FB } & Ausente (-) & $7(100,0)$ & $\mathrm{O}(\mathrm{O}, \mathrm{O})^{*}$ & $\mathrm{O}(0,0)^{*}$ & 7 \\
\hline & & Presente (+) & $90(55,2)^{5}$ & $44(27,0)^{* \S}$ & $29(17,8)^{\star \S}$ & 163 \\
\hline \multirow{4}{*}{ TE } & \multirow{2}{*}{ FM } & Ausente (-) & $85(67,4)$ & $40(31,7)^{*}$ & $1(0,8)^{\star \#}$ & 126 \\
\hline & & Presente (+) & $12(27,3)^{\S}$ & $4(9,0)^{\S}$ & $28(63,6)^{\star \S}$ & 44 \\
\hline & \multirow{2}{*}{ FB } & Ausente (-) & $2(66,7)$ & $\mathrm{O}(0,0)$ & $1(33,3)$ & 3 \\
\hline & & Presente $(+)$ & $95(56,9)^{\S}$ & $44(26,3)^{*}$ & $28(16,8)$ & 167 \\
\hline \multirow{2}{*}{\multicolumn{2}{|c|}{ Schöber }} & Ausente (-) & $78(63,4)$ & $24(19,5)^{*}$ & $21(17,1)^{*}$ & 123 \\
\hline & & Presente (+) & $19(40,4)$ & $20(42,6)^{\star \S}$ & $8(17,0)^{* \#}$ & 47 \\
\hline
\end{tabular}

G1: atletas da faixa etária de 11 a 14 anos de idade; G2: atletas da faixa etária de 15 a 18 anos; G3: atletas adultos; TD: Teste de Thomas para Membro Inferior Direito; TE: Teste de Thomas para Membro Inferior Esquerdo; FM: Flexores de Quadril Monoarticulares; FB: Flexores de Quadril Biarticulares. Comparações horizontais (grupo): * $\mathrm{p}<0,05$ versus $\mathrm{G1}$; ${ }^{*} \mathrm{p}<0,05$ versus $\mathrm{G} 2$ Comparações verticais: ${ }^{\$} \mathrm{p}<0,05$ versus Ausente (-); Teste de Goodman para contraste entre e dentro de populações multinomiais $(p<0,05)$ 
lombar foi particularmente importante no G1; a ocorrência de teste de Schöber positivo se associou com probabilidade três vezes maior de LD (OR=2,80 com IC 0,99-7,89; $\mathrm{p}=0,047$ ).

Em relação à extensibilidade da cadeia muscular posterior, verificou-se que G1 e G2 revelaram maior prevalência de alterações posturais na Postura 1. Na Postura 2, o G1 mostrou alteração em 81 casos $(98,8 \%)$ e o G3 reportou 16 ocorrências (72,7\%; $\mathrm{p}<0,05)$ " para $\mathrm{Na}$ Postura 2, o G1 mostrou alteração em 81 casos $(98,8 \%)$ e o G3 reportou 16 ocorrências (72,7\%; $\mathrm{p}<0,05$; Tabela 3$)$.

Entre os adultos, a retração segmentar na Postura 2 se associou com chance 14 vezes maior de $\mathrm{LD}(\mathrm{OR}=14,0$; IC 1,4-137,3; $\mathrm{p}=0,023)$. A presença de desordens na Postura 1 se relacionou com probabilidade 4,88 vezes maior de instalação de LD no $\mathrm{G} 3(\mathrm{OR}=4,88$; IC 0,7830,30; $\mathrm{p}=0,089$ ) (Tabela 3).

\section{DISCUSSÃO}

O presente trabalho se propôs a analisar a flexibilidade articular, extensibilidade muscular e a prevalência de LD em praticantes de futebol, caracterizando-as segundo a idade. Como objetivo adicional, buscou-se analisar a associação entre essas características e a incidência de LD. O delineamento do estudo justifica-se pela possibilidade de conferir subsídios à atuação profissional na profilaxia de fatores de risco intrínseco e tratamento de LD, contribuindo para a evolução do desempenho físico, tático e técnico de praticantes de futebol nas principais categorias.

O G2 revelou maiores índices de flexibilidade de músculos posteriores de coxa. Estes achados se associaram com maior prevalência $(42,6 \%)$ de inflexibilidade lombar. O G2 integra atletas com maior histórico de prática de futebol. Portanto, os valores de flexibilidade de coxa posterior poderiam resultar da interação entre

Tabela 3. Distribuição absoluta e relativa (\%) de retrações da cadeia mestra posterior segundo postura e faixa etária

\begin{tabular}{lcccc}
\multirow{2}{*}{ Grupos } & \multicolumn{2}{c}{ Postura 1 } & \multicolumn{2}{c}{ Postura 2} \\
\cline { 2 - 5 } & Ausência (-) & Presença (+) & Ausência (-) & Presença (+) \\
G1 & $16(16,5)$ & $81(83,5)^{\star}$ & $2(2,1)$ & $95(97,9)^{\star}$ \\
G2 & $12(27,3)$ & $32(72,7)^{\star}$ & $2(4,5)$ & $42(95,5)^{\star}$ \\
G3 & $8(38,1)$ & $21(61,9)$ & $8(38,1)^{\S}$ & $21(61,1)^{\star \star \S}$
\end{tabular}

G1: atletas da faixa etária de 11 a 14 anos de idade; G2: atletas da faixa etária de 15 a 18 anos; G3: atletas adultos. Comparações horizontais (grupo): * $\mathrm{p}<0,05$ versus ausência de retrações. Comparações verticais (grupo): ${ }^{\mathrm{p}}<0,05$ versus $\mathrm{G1}$; $\mathrm{*}<<0,05$ versus $\mathrm{G} 2$; Teste de Goodman para contraste entre e dentro de populações multinomiais $(p<0,05)$ fatores intrínsecos, comuns à maturação cronológica ${ }^{15}$, e atributos extrínsecos, relacionados ao futebol. Assim, efeitos derivados do crescimento ósseo e muscular, aliados ao aprimoramento musculoesquelético imposto pela prática regular de treinamento desportivo especializado podem ter culminado em aumento da flexibilidade muscular de músculos posteriores da coxa. Por analogia, é possível que tais fatores tenham desencadeado adaptações lombares, em razão de maior demanda pela estabilização segmentar de tronco e pelve, necessária para execução de gestual motor específico do futebol, incluindo-se corrida, chutes e pivoteios ${ }^{4,5}$. Ainda que meramente especulativo para o $\mathrm{G} 2$, tal preceito constitui importante suporte para a associação entre retração lombar e ocorrência de LD em G1 $(\mathrm{OR}=2,8)$. Este grupo é constituído por atletas iniciantes, com histórico de treinamento mais breve, sendo possível que essas respostas adaptativas sejam mais acentuadas no início da sistematização do treinamento físico desportivo, podendo culminar, mais tarde, em probabilidade aumentada de LD.

Além disso, é muito provável que as demandas físico-motoras listadas se associem com retração de flexores biarticulares de quadril, pois todos os grupos apresentaram altos índices deste distúrbio muscular. A prevalência de retração foi progressivamente ampliada com a idade e com o histórico de treinamento, culminando em taxas de 96,6 a $100 \%$ em atletas adultos. Devido à maior demanda contrátil de músculos flexores de quadril durante gestos específicos, a retração desse grupo muscular configura um achado muito comum entre jogadores de futebol ${ }^{10,11}$, podendo culminar em hiperlordose lombar, anteversão pélvica e retesamento de músculos posteriores de $\operatorname{coxa}^{20}$. Em particular, ao ingressar precocemente em centros de formação desportiva, atletas jovens são submetidos a solicitações de força e potência, não contrabalançadas por exercícios específicos de flexibilidade ${ }^{10}$. Com isso, pode-se dizer que as exigências específicas do futebol conduzem a desequilíbrios entre grupos musculares agonistas e antagonistas, favorecendo a ocorrência de mudanças posturais ${ }^{9,12}$. Aliados a erros na técnica de execução dos movimentos, tais fatores podem consistir em importantes causas de lesões musculares ${ }^{12}$, mostradas como as principais LD no futebol ${ }^{23}$.

Embora a prevalência de retração da cadeia mestra posterior tenha se reduzido com o envelhecimento, tal resposta foi um importante achado. É possível que estes resultados decorram não só do futebol, como também de disparidades no desenvolvimento osteomioarticular, 
comuns à adolescência. Os mecanismos de sobrecarga decorrente da prática sistemática de exercício físico se associam com aumento da densidade mineral óssea ${ }^{26}$. Tais respostas, entretanto, são mais efetivas quando a solicitação física se desenvolve simultaneamente com a velocidade máxima de maturação óssea biológica, que se dá durante a puberdade ${ }^{26,27}$. Além disso, os efeitos osteogênicos do exercício são também direcionados pela frequência e intensidade de sobrecarga, sendo que solicitações físicas de força e potência muscular em situações de impacto, como vigente no futebol ${ }^{4}$, aceleram o desenvolvimento ósseo ${ }^{26}$. Em contraste, estas solicitações geralmente culminam em hipertrofia e encurtamento muscular ${ }^{9}$. Tal desarmonia entre crescimento ósseo e maturação muscular pode ter sustentado a menor extensibilidade da cadeia mestra posterior em G1 e G2, constituindo importante componente etiológico na promoção de agravos específicos da adolescência, como a doença de Osgood-Schlatter e a doença de Sever ${ }^{28}$.

Embora amenizada no G3, a presença de encurtamento global da cadeia posterior foi marcadamente importante, resultando em 14 vezes mais chances de instalação de LD na vida adulta. A repetição de certos tipos de atividade com posições e movimentos habituais, aliada ao período e à sobrecarga de treinamento, provoca um processo de adaptação orgânica que resulta em efeitos deletérios para a postura, com alto potencial de desequilíbrio muscular ${ }^{13,21}$, o que é sustentado pelo ampliado histórico de treinamento do G3. Ademais, os gestos específicos do esporte e os erros na técnica de execução dos movimentos podem aumentar a prevalência de $\mathrm{LD}^{6}$.

Não obstante, a natureza transversal deste trabalho confere potencial apenas especulativo para os presentes achados. Portanto, ressalta-se a necessidade de estudos adicionais com o intuito de se confirmar quais os fatores etiológicos são mais atuantes na ocorrência de LD segundo a idade.

\section{CONCLUSÕES}

Os resultados permitem concluir que atletas adultos têm maior incidência e prevalência de LD, que decorre, principalmente, de retrações da cadeia mestra posterior. Porém, os índices de retração da coluna lombar foram particularmente importantes na fisiopatologia de LD entre adolescentes, sugerindo que uma possível interação entre atributos intrínsecos e extrínsecos se relacione na ocorrência de agravos em jovens atletas.

\section{AGRADECIMENTOS}

Nossos agradecimentos à Direção e aos funcionários do Clube Esportivo Nova Esperança (CENE), por todo o auxílio prestado para a realização do presente estudo, e ao Sr. Sérgio Roberto de Oliveira, pelas contribuições técnicas finais.

\section{REFERÊNCIAS}

1. Rocha HPA, Bartholo TL, Melo LBS, Soares AJG. Jovens esportistas: profissionalização no futebol e a formação na escola. Motriz Rev Educ Fis. 2011;17(2):252-63.

2. Soares AJG, Melo LBS, Costa FR, Bartholo TL, Bento JO. Jogadores de futebol no Brasil: mercado, formação de atletas e escola. Rev Bras Ciênc Esporte. 2011;33(4):905-21.

3. Ribeiro RN, Vilaça F, Oliveira HU, Vieira LS, Silva AA. Prevalência de lesões no futebol em atletas jovens: estudo comparativo entre diferentes categorias. Rev Bras Educ Fís Esp. 2007;21(3):189-94.

4. Manning MR, Levy RS. Soccer. Phys Med Rehabil Clin N Am. 2006:17(3):677-95

5. Braz TV, Spigolon LMP, Borin JP. Caracterização dos meios e métodos de influência prática no treinamento em futebolistas profissionais. Rev Bras Ciênc Esporte. 2012;34(2):495-511.

6. Bahr R, Krosshaug T. Understanding injury mechanisms: a key component of preventing injuries in sport. $\mathrm{Br} J$ Sports Med. 2005:39(6):324-9.

7. Schmikli SL, de Vries WR, Inklaar H, Backx FJG. Injury prevention target groups in soccer: injury characteristics and incidence rates in male junior and senior players. J Sci Med Sport. 2011;14(3):199-203.

8. Zanuto EAC, Harada H, Filho LRAG. Análise epidemiológica de lesões e perfil físico de atletas do futebol amador na região do oeste paulista. Rev Bras Med Esporte. 2010;16(2):116-20.

9. Alter MJ. Science of flexibility. 2. ed. Champaign: Human Kinetics; 1996.

10. Bertolla F, Baroni BM, Junior ECPL, Oltramari JD. Efeito de um programa de treinamento utilizando o método Pilates ${ }^{\circledR}$ na flexibilidade de atletas juvenis de futsal. Rev Bras Med Esporte. 2007:13(4):222-6.

11. Kleinpaul JF, Mann L, Santos SG. Lesões e desvios posturais na prática de futebol em jogadores jovens. Fisioter Pesq. 2010;17(3):236-41.

12. Veiga PHA, Daher CRM, Morais MFF. Alterações posturais e flexibilidade da cadeia posterior nas lesões em atletas de futebol de campo. Rev Bras Ciênc Esporte. 2011;33(1):235-48.

13. Ribeiro CZP, Akashi PMH, Sacco IMN, Pedrinelli A. Relationship between postural changes and injuries of the locomotor system in indoor soccer athletes. Rev Bras Med Esporte 2003;9(2):98-103.

14. Cain LE, Nicholson LL, Adams RD, Burns J. Foot morphology and foot/ankle injury in indoor football. J Sci Med Sport. 2007:10(5):311-9. 
15. Gallahue DL, Ozmun JC. Compreendendo o desenvolvimento motor: bebês, crianças, adolescentes e adultos. São Paulo: Phorte Editora; 2005.

16. Göeken LN, Hof L. Instrumental straight-leg raising: Results in healthy subjects. Arch Phys Med Rehabil. 1993;74(4):194-203.

17. Gajdosik RL. Passive extensibility of skeletal muscle: review of the literature with clinical implications. Clin Biomech (Bristol, Avon). 2001; 16(2):87-101.

18. Leite N, Aguiar-Junior RP, Cieslak F, Ishiyama M, Milano GE, Stefanello JMF. Perfil da aptidão física dos praticantes de Le Parkour. Rev Bras Med Esporte. 2011;17(3):198-201.

19. Macedo CSG, Souza PR, Alves PM, Cardoso JR. Estudo da validade e confiabilidade intra e interobservador da versão modificada do teste de Schöber modificado em indivíduos com lombalgia. Fisioter Pesq. 2009;16(3):233-8.

20. Kendall HO, Kendall FP, Wadsmorth GE. Músculos: provas e funções. São Paulo: Manole, 1995.

21. Neto Junior J, Pastre CM, Monteiro HL. Alterações posturais em atletas brasileiros do sexo masculino que participaram de provas de potência muscular em competições internacionais. Rev Bras Med Esporte. 2004;10(3):195-8.
22. Pastre CM, Filho GC, Monteiro HL, Junior JN, Padovani CR. Lesões desportivas na elite do atletismo brasileiro: estudo a partir de morbidade referida. Rev Bras Med Esporte. 2005;11(1):43-7.

23. Silveira KP, Assunção VHS, Guimarães-Júnior N, Miziara-Barbosa S, Santos MLM, Christofoletti G, et al. Perfil nosográfico de lesões desportivas no futebol segundo faixa etária. Rev Bras Cineantropom Desempenho Hum. 2013 (no prelo). 15(4):476-83.

24. Bennell KL, Crossley K. Musculoskeletal injuries in track and field: incidence, distribution and risk factors. Aust J Sci Med Sport. 1996;28(3):69-75.

25. Goodman LA. On simultaneous confidence intervals for multinomial proportions. Technometrics. 1965;7(2):247-54.

26. Silva CC, Teixeira AS, Goldberg TB. O esporte e suas implicações na saúde óssea de atletas adolescentes. Rev Bras Med Esporte. 2003;9(6):426-32.

27. Broderick CR, Winter GJ, Allan RM. Sport for special groups. Med J Aust. 2006:184(6):297-302.

28. Lau LL, Mahadev A, Hui JHP. Common lower limb sports-related overuse injuries in young athletes. Ann Acad Med Singapore. 2008;37(4):315-9. 